\title{
HOPF'S LEMMA FOR A CLASS OF SINGULAR/DEGENERATE PDE-S
}

\author{
Hayk Mikayelyan and Henrik Shahgholian \\ Xi'an Jiaotong-Liverpool University, Department of Mathematical Sciences \\ Ren Ai Lu 111, 215123 Suzhou, Jiangsu Province, P. R. China; Hayk.Mikayelyan@xjtlu.edu.cn \\ Royal Institute of Technology (KTH), Department of Mathematics \\ 10044 Stockholm, Sweden; henriksh@math.kth.se
}


for a class of singular/degenerate PDE-s, including $p$-Laplacian. Using geometric properties of levels sets for harmonic functions in convex rings, we construct sub-solutions to our equations that play the role of a barrier from below. By comparison principle we then conclude Hopf's lemma.
\end{abstract}

\section{Introduction}

1.1. Background. Construction of barriers is among many important and fundamental tools in the analysis of partial differential equations. Barriers may be used to quantify the behavior of solutions to partial differential equations close to the boundary of the bulk domain. One of many consequences of barriers is the Hopf's boundary point lemma (or principle), originated in a paper by Hopf [Hopf], and later developed in many diverse forms. Hopf's boundary principle states that a super-solution to a partial differential equation with a minimum value at a boundary point, must increase (away from this boundary point) with a given quantity that is dictated by the ingredients of the problem. In particular it means that the function increases in a linear fashion away from its minimum value on the boundary, provided the boundary is smooth enough.

This boundary point lemma has been successfully developed and applied to many different problems. The main tool in proving it uses a simple barrier and the maximum principle. Therefore, the Hopf's lemma can be deduced once we have a barrier. In domains with enough boundary regularity (say $C^{2}$ ) one can actually construct simple (spherical) barriers for second order uniformly elliptic equation; K. Widman proved the result for domains of Liapunov-Dini type (see below). Hopf's lemma for other operatros, in particular the $p$-Laplacian, has been shown by Tolksdorf (see [T, Proposition 3.2.1]) for domains whose boundary has interior touching ball.

It would be interesting to see whether ideas in this paper can be developed to yield further results on related type of problems. E.g., the strong comparison principle for singular/degenerate operators is still unknown, even though there are partial results in the literatures (see [T, Dam]).

doi:10.5186/aasfm.2015.4033

2010 Mathematics Subject Classification: Primary 35J25, 35J70.

Key words: Partial differential equations, regularity. 
In this paper we consider Hopf's lemma, in certain $C^{1, \text { Dini }}$-type ${ }^{1}$ domains, for the following type of operators

$$
\Delta_{H} u:=\operatorname{div}(H(|\nabla u|) \nabla u),
$$

where $H(t)=t^{-1} h(t), h(0)=0$ and $h(t)$ is a monotone increasing continuous function, satisfying some structural conditions (see Section 1.3).

1.2. Main result. The main result of this paper is the following extension of Widman's result to a wider class of operators (1), for which $p$-Laplacian is a particular case, in Liapunov-Dini domains with convex-Dini modulus of continuity (see Definition 5 below). A simple consequence of our result (Remark 8) is the boundary Harnack principle for $H$-harmonic functions in domains with convex-Dini boundary. For $p$-Laplacian case the boundary Harnack principle was settled in Lipschitz domains in [LN]. It seems plausible that the techniques in [LN] can be applied to derive similar results as ours, but that would require a detailed technical study and adaptation of a deep and highly technical analysis to our case. In light of this technical machinery our approach seems very easy and almost elementary.

Theorem 1. Assume $u$ is a weak solution of equation (1) in the domain $D$. Further assume $0 \in \partial D, \partial D$ satisfies the inner convex-Dini condition at 0 (see Definition 5 below) and

$$
u(x)>u(0) \text { for all } x \in D .
$$

Then there exist positive constants $r_{0}$ and $c$ such that

$$
\max _{B_{r} \cap D} u(x)-u(0)>c r,
$$

for $0<r<r_{0}$.

The proof of this theorem shall need several tools and definitions that will be introduced in the next subsections. The proof then will be given in Section 2 .

1.3. Structural conditions. In order to formulate the main result we shall need a few definitions, and notations. For simplicity we shall call the weak solutions of (1) $H$-harmonic functions. This type of operators arise in applications dealing with flows where the flow-rate is proportional to

$$
H(|\nabla u|) \nabla u=h(|\nabla u|) \frac{\nabla u}{|\nabla u|} .
$$

Let us now list all the assumptions we impose on $h$, that can formally be divided into three groups:

- physical (see equations (2)),

- coercive (see equation (4), or (5)),

- technical (see equation (6)).

The following three conditions on $h$ have physical nature.

- $h(0)=0$ : with vanishing gradient flow vanishes;

(2) • monotonicity of $h(t)$ : larger gradient $\Longrightarrow$ more flow;

- $H$ depends only on $|\nabla u|$ : isotropy with respect to the position and direction.

\footnotetext{
${ }^{1}$ The additional condition we impose on the Dini modulus of continuity $\epsilon(t)$ is that the function $t \epsilon(t)$ is convex (more discussion in Section 1.4).
} 
Equation (1) is the Euler-Lagrangian of the functional

$$
I(v)=\int_{D} F(|\nabla v|) d x
$$

where $F(t)=\int_{0}^{t} h(\tau) d \tau, F \in C^{1}([0, \infty)), F(0)=F^{\prime}(0)=0$ and $F$ is strictly convex.

The coercivity condition is needed to assure the existence of the solution (minimizer). It is well known that this problem is, in general, ill-posed if $h$ is bounded. The best illustration is the minimal surface equation

$$
\operatorname{div}\left(\frac{\nabla u}{\sqrt{1+|\nabla u|^{2}}}\right)
$$

in the annulus $B_{2} \backslash B_{1}$, with boundary data 0 on $\partial B_{2}$ and $M$ on $\partial B_{1}$. For large enough $M$ the catenoid cannot reach the level $M$ without leaving the domain $B_{2} \backslash B_{1}$ and the equation has no solution in $W^{1,1}(D)$. To avoid this we can impose the rather strong condition

$$
c t^{p-1}<h(t)<C t^{p-1}
$$

for some $p>1$, which makes the application of the direct methods of the calculus of variations in the Sobolev space $W^{1, p}(D), p>1$, possible (see [D]). In the special case $H(t)=t^{p-2}$ we obtain the $p$-Laplacian.

Alternatively we can impose a weaker coercivity condition and work in SobolevOrlicz spaces $W^{1, F}(D)$. Let us shortly introduce these spaces following $[\mathrm{RR}]$ (see also [RR1]). The Orlicz norm is defined as follows

$$
\|u\|_{F}=\min \left\{M \mid \int_{D} F\left(\frac{|u|}{M}\right) d x \leq F(1)\right\} .
$$

This norm defines the Banach space $L^{F}(D)$. If we now denote by $g$ the inverse function of $h$ and define the Legendre transform of $F$ by

$$
F^{*}(t)=\int_{0}^{t} g(\tau) d \tau
$$

then assuming $h(1)=1$ one can easily prove using Young's inequality the generalization of Hölder's inequality

$$
\int_{D} u v d x \leq\|u\|_{F}\|v\|_{F^{*}}
$$

If both $F$ and its Legendre transform $F^{*}$ satisfy the so-called $\Delta_{2}$ condition, i.e., there exists $t_{0}>0, C_{0}>0$ such that

$$
F(2 t) \leq C_{0} F(t) \text { and } F^{*}(2 t) \leq C_{0} F^{*}(t) \text { for } t>t_{0},
$$

then

$$
\left(L^{F}(D)\right)^{*}=L^{F^{*}}(D)
$$

and, in particular, $L^{F}(D)$ is reflexive, since $\left(F^{*}\right)^{*}=F$ [RR, Theorem 10, p. 112].

Now analogously we can define the Sobolev-Orlicz space $W^{1, F}(D)$ by the SobolevOrlicz norm as sum of Orlicz norms of $u$ and $|\nabla u|$. Under $\Delta_{2}$ condition $W^{1, F}(D)$ will be reflexive and we can apply the direct methods of the calculus of variations.

The technical condition is presented in the following remark. 
Remark 1. In our proof the function

$$
R(t)=\frac{F^{\prime \prime}(t)}{F^{\prime}(t)}
$$

plays an important role and we need the following technical condition on $R$. We assume that for every positive, monotone increasing, bounded function $c(s)$ in $\mathbf{R}^{+}$, $0<c<c(s)<C<\infty$, there exist constants $\alpha>0$ and $\beta>0$, depending on function $R$, and constants $c$ and $C$, such that

$$
\int_{t}^{T} R(c(s) s) d s \geq \alpha \int_{t}^{T} R(\beta s) d s .
$$

Condition (6) is satisfied for more or less any "reasonable" function $R=F^{\prime \prime} / F^{\prime}$. For monotone decreasing $R$ one can take $\alpha=1, \beta=C$ (this covers the case $F(t)=t^{p}$, $p>1$ ). The authors think that it is easier to check the condition (6) for a given function $F$, than to try to introduce a broad class of functions satisfying it.

Definition 2. ( $H$-potential) For two convex domains $K_{1} \Subset K_{2}$ we call the minimizer $u$ of

$$
J(v)=\int_{K_{2} \backslash K_{1}} F(|\nabla v|) d x
$$

in the class of functions $\left\{v \in W_{0}^{1, F}\left(K_{2}\right) \mid v \equiv 1\right.$ on $\left.K_{1}\right\}$ an $H$-potential (see [RR]).

1.4. Liapunov-Dini boundary. In the case of harmonic functions $\left(F(t)=t^{2}\right)$ Widman [W] using the Green representation was able to prove a Hopf-type result for domains with Liapunov-Dini boundary (see below). Moreover, the following estimates for the second derivatives of the solution $v$ of uniformly elliptic equation with Hölder continuous coefficients have been proved as well (see equation (2.4.1) in $[\mathrm{W}])$

$$
\left|D^{2} v(x)\right| \leq C_{D} \frac{\epsilon(\delta(x))}{\delta(x)},
$$

where $\epsilon(t)$ is a Dini modulus of continuity (see (3) below) and $\delta(x)$ is the distance of the point $x$ from the boundary. It is also shown that $C^{1, \text { Dini }}$ regularity is necessary for Hopf lemma in axially symmetric domains (see Remark 1 in [W]).

Since there is no Green representation for $p$-harmonic functions it is not possible to repeat Widman's direct estimates of the function's growth. Our proof is based on barrier construction and works for the general operator $\Delta_{H}$ under some regularity assumptions on the boundary.

Let us present the definition of Liapunov-Dini surface following [W].

Definition 3. A modulus of continuity $\epsilon(r) \searrow 0$ as $r \rightarrow 0$ is called Dini modulus of continuity if $\int t^{-1} \epsilon(t) d t<\infty$.

Definition 4. A Liapunov-Dini surface $S$ is a closed, bounded $(n-1)$-dimensional surface satisfying the following conditions:

(a) At every point of $S$ there is a uniquely defined tangent hyper-plane, and thus also a normal.

(b) There exits a Dini modulus of continuity $\epsilon(t)$ such that if $\beta$ is the angle between two normals, and $r$ is the distance between their foot points, then the inequality $\beta \leq \epsilon(r)$ holds. 
(c) There is a constant $\rho_{S}>0$ such that for any point $x \in S$, any line parallel to the normal at $x$ meets $S \cap B_{\rho_{s}}(x)$ at most once.

In simple words the above definitions says, that the surface $S$ is locally the graph of a $C^{1, \text { Dini }}$ function in a ball of fixed radius.

Since in general the function $t \epsilon(t)$ is not convex we introduce a sub-class of Dini modules of continuity as follows.

Definition 5. A Dini modulus of continuity $\epsilon(r)$ is called convex-Dini if the function $t \epsilon(t)$ is convex.

Note that domains with $C^{1, \alpha}$ boundary are convex-Dini. The introduction of such a sub-class is necessary because our proof relies on the construction of barriers in convex rings with $C^{1 \text {,Dini }}$ boundary. It it in general not true that for any Dini modulus of continuity $\epsilon(t)$ there is another Dini modulus of continuity $\tilde{\epsilon}(t) \geq \epsilon(t)$ such that $t \tilde{\epsilon}(t)$ is convex.

Remark 6. If the Dini modulus of continuity is convex-Dini then a domain $D$ with Liapunov-Dini boundary satisfies a kind of inner (outer) convex $C^{1 \text {,Dini }}$ condition in the following sense: There exists a convex Liapunov-Dini domain $K$ such that for any point $x_{0} \in \partial D$ there exists a translation and rotation $K_{x_{0}}$ of the domain $K$ satisfying

$$
K_{x_{0}} \subset D, \quad\left(K_{x_{0}} \subset \mathbf{R}^{n} \backslash D\right) \quad \text { and } \quad \partial K_{x_{0}} \cap \partial D=\left\{x_{0}\right\} .
$$

Moreover, we can take

$$
K=K_{r_{D}}=B_{r_{D}}\left(\left(0, \ldots, 0, r_{D}\right)\right) \cap\left\{x\left|x_{n}>2\right| x^{\prime} \mid \epsilon\left(\left|x^{\prime}\right|\right)\right\},
$$

where $x=\left(x^{\prime}, x_{n}\right), r_{D}<\rho_{\partial D} / 2$ and $\epsilon$ is the convex-Dini modulus of continuity. Without loss of generality (if necessary by modifying $\epsilon$ at "corners" with $\partial B_{r_{D}}((0, \ldots$, $\left.\left.0, r_{D}\right)\right)$ ) we can assume that $K$ has smooth boundary. Let us also observe that for any $a>0$ by taking $r_{D}$ small enough we can have

$$
B_{(1-a) r_{D}}\left(\left(0, \ldots, 0, r_{D}\right)\right) \Subset K .
$$

Let us assume that for $r_{D}$

$$
B_{\frac{3}{4} r_{D}}\left(\left(0, \ldots, 0, r_{D}\right)\right) \Subset K .
$$

In the sequel we will use as barriers the $H$-potentials in the convex rings

$$
K \backslash B_{r_{D} / 2}\left(\left(0, \ldots, 0, r_{D}\right)\right) \text { and } B_{3 r_{D}}\left(\left(0, \ldots, 0,-r_{D}\right)\right) \backslash(-K),
$$

where $-K$ is obtained from $K$ by symmetry with respect to the origin. We will refer to convex rings (10) as inner and out convex rings.

\section{Proof of main result}

Without loss of generality we can assume that the outer normal of $\partial D$ a the origin is $(0, \ldots, 0,-1)$. Since for the solutions of (1) we have the maximum principle (see Appendix II) we need to construct a barrier in the inner convex ring from (10).

Actually we will construct barriers in arbitrary convex ring $K_{2} \backslash K_{1}$, where $K_{1} \Subset$ $K_{2}$ are two convex domains with Liapunov-Dini boundary.

From the Hopf lemma for harmonic functions ([W]) we know that if $\Delta w=0$ in $K_{2} \backslash K_{1}$, with boundary values $w=0$ on $\partial K_{2}$ and $w=1$ on $\partial K_{1}$ then $\nabla w \neq 0$ on 
$\partial\left(K_{2} \backslash K_{1}\right)$. Now we will prove the existence of a convex, smooth, monotone increasing function $f:[0,1] \rightarrow[0,1], f(0)=0, f(1)=1, f^{\prime}(0)>0, f^{\prime}(1)<\infty$ such that

$$
\Delta_{H} f(w) \geq 0
$$

in $K_{2} \backslash K_{1}$. This will mean that the function $f(w)$ is a subsolution for $\Delta_{H}$, has non-vanishing gradient at any boundary point and thus can be used as a barrier.

We start by computing

$$
\Delta_{H} f(w)=H(|\nabla f(w)|) \Delta f(w)+\frac{H^{\prime}(|\nabla f(w)|)}{|\nabla f(w)|} \Delta_{\infty} f(w),
$$

where $\Delta_{\infty} u=\nabla u D^{2} u \nabla u$ is the $\infty$-Laplace operator. Using

$$
\Delta f(w)=f^{\prime}(w) \Delta w+f^{\prime \prime}(w)|\nabla w|^{2}=f^{\prime \prime}(w)|\nabla w|^{2},
$$

and

we arrive at

$$
\Delta_{\infty} f(w)=\left(f^{\prime}(w)\right)^{3} \Delta_{\infty} w+\left(f^{\prime}(w)\right)^{2} f^{\prime \prime}(w)|\nabla w|^{4}
$$

$$
\begin{aligned}
\Delta_{H} f(w)= & H\left(f^{\prime}(w)|\nabla w|\right) f^{\prime \prime}(w)|\nabla w|^{2} \\
& +\frac{H^{\prime}\left(f^{\prime}(w)|\nabla w|\right)}{f^{\prime}(w)|\nabla w|}\left[\left(f^{\prime}(w)\right)^{3} \Delta_{\infty} w+\left(f^{\prime}(w)\right)^{2} f^{\prime \prime}(w)|\nabla w|^{4}\right] .
\end{aligned}
$$

We thus need to find a function $f$, such that $f^{\prime}(t)>0$ for $t \in[0,1]$ and $\Delta_{H} f(w) \geq 0$. To comply with the latter we need (see (12))

$$
\begin{aligned}
& f^{\prime \prime}(w)|\nabla w|^{2}\left[H\left(f^{\prime}(w)|\nabla w|\right)+f^{\prime}(w)|\nabla w| H^{\prime}\left(f^{\prime}(w)|\nabla w|\right)\right] \\
& \geq-\frac{H^{\prime}\left(f^{\prime}(w)|\nabla w|\right)}{f^{\prime}(w)|\nabla w|}\left(f^{\prime}(w)\right)^{3} \Delta_{\infty} w
\end{aligned}
$$

which after substitution $H^{\prime}(t)=\frac{F^{\prime \prime}(t)}{t}-\frac{F^{\prime}(t)}{t^{2}}$ and $H(t)+t H^{\prime}(t)=F^{\prime \prime}(t)$ simplifies to

$$
f^{\prime \prime}(w) \geq\left(\frac{F^{\prime}\left(f^{\prime}(w)|\nabla w|\right)}{F^{\prime \prime}\left(f^{\prime}(w)|\nabla w|\right)}-f^{\prime}(w)|\nabla w|\right)|\nabla w|^{-5} \Delta_{\infty} w .
$$

Let us note that

$$
0=\Delta w=\partial_{\nu \nu} w-(n-1) \kappa \partial_{\nu} w,
$$

where $\nu$ is the unit vector in the direction of $\nabla w$ and $\kappa$ is the mean curvature of the level set; here we have used that the level sets of a positive harmonic potential are smooth. From this we conclude

$$
\Delta_{\infty} w=\left(\partial_{\nu} w\right)^{2} \partial_{\nu \nu} w=(n-1) \kappa|\nabla w|^{3} .
$$

Since the level sets of a harmonic potential in a convex ring are convex (see [L]), the mean curvature and thus $\Delta_{\infty} w$ is positive near the boundary. This along with $f^{\prime}(w)>0$ and (13) (which is yet to be proven) implies that it is enough to find a function $f$ such that

$$
f^{\prime \prime}(w) R\left(f^{\prime}(w)|\nabla w|\right) \geq|\nabla w|^{-5} \Delta_{\infty} w
$$

where $R(t)=\frac{F^{\prime \prime}(t)}{F^{\prime}(t)}$.

The Hopf lemma proved in [W] for harmonic functions yields

$$
0<c<|\nabla w(x)|<C<\infty,
$$


where the constants $c$ and $C$ depend only on the convex ring. Using this we easily obtain

$$
c \min (w(x), 1-w(x)) \leq \delta(x) \leq C \min (w(x), 1-w(x))
$$

and together with (8)

$$
\begin{aligned}
|\nabla w|^{-5}\left|\Delta_{\infty} w\right| & \leq c^{-3}\left|D^{2} w\right| \leq c^{-3} C_{D} \min \frac{\epsilon(\delta(x))}{\delta(x)} \\
& \leq c^{-3} C_{D} \frac{\epsilon(C \min (w, 1-w))}{c \min (w, 1-w)}=: \zeta(w),
\end{aligned}
$$

where $\zeta(t) \in L^{1}([0,1])$ depend only on the convex ring.

In order to have (15) we need to construct a function $f$ such that

$$
f^{\prime \prime}(w) R\left(f^{\prime}(w)|\nabla w|\right) \geq \zeta(w) .
$$

For any $x \in K_{2} \backslash K_{1}$ let us denote by $\ell_{x}$ the gradient flow line of $w$ which contains $x$. Let us parametrize the curve $\ell_{x}$ by $w \in[0,1]$. We can now integrate $(17)$ on any $\ell_{x}$ in parameter $w$

$$
\int_{w_{1}}^{w_{2}} f^{\prime \prime}(w) R\left(f^{\prime}(w)|\nabla w|\right) d w \geq \int_{w_{1}}^{w_{2}} \zeta(w) d w
$$

Observe that since the level sets of $w$ are convex the function $|\nabla w|$ on $\ell_{x}$ as a function of $w$ are monotone increasing (see equation (14)), but on the other hand we know that it is bounded by (16). Thus we can apply our technical condition (6)

$$
\int_{w_{1}}^{w_{2}} f^{\prime \prime}(w) R\left(f^{\prime}(w)|\nabla w|\right) d w=\int_{f^{\prime}\left(w_{1}\right)}^{f^{\prime}\left(w_{2}\right)} R(c(s) s) d s \geq \alpha \int_{f^{\prime}\left(w_{1}\right)}^{f^{\prime}\left(w_{2}\right)} R(\beta s) d s,
$$

where $s=f^{\prime}(w)$ and the function $c(s)=|\nabla w|(s)>0$ is a monotone function such that $c<c(s)<C$.

If we now construct a function $f$ such that

$$
\alpha \int_{f^{\prime}\left(w_{1}\right)}^{f^{\prime}\left(w_{2}\right)} R(\beta s) d s \geq \int_{w_{1}}^{w_{2}} \zeta(w) d w
$$

for all $0<w_{1}<w_{2}<1$, then for this function $f$ the inequality (18) will be satisfied for all gradient flow lines $\ell_{x}$ and thus the inequality (17) will be satisfied everywhere in $K_{2} \backslash K_{1}$, and we would be done.

Since $F^{\prime}(0)=0$ and $F^{\prime}(\infty)=\infty$, the function $R(t)=\frac{F^{\prime \prime}(t)}{F^{\prime}(t)}$ is not integrable near zero and at $+\infty$, due to

$$
\int_{t}^{T} R(\tau) d \tau=\log \frac{F^{\prime}(T)}{F^{\prime}(t)}
$$

As $w_{1} \rightarrow 0$ and $w_{2} \rightarrow 1$ the right hand side of (19) remains bounded $\left(\zeta \in L^{1}(0,1)\right.$ ) and we can write

$$
\alpha \int_{f^{\prime}\left(w_{1}\right)}^{f^{\prime}\left(w_{2}\right)} R(\beta s) d s=\frac{\alpha}{\beta}\left(\log F^{\prime}\left(\beta f^{\prime}\left(w_{2}\right)\right)-\log F^{\prime}\left(\beta f^{\prime}\left(w_{1}\right)\right)\right)=\int_{w_{1}}^{w_{2}} \zeta(w) d w .
$$

Now we can take $f^{\prime}(0)=m>0$ and construct

$$
f^{\prime}(w)=\beta^{-1} g\left(F^{\prime}(\beta m) e^{\frac{\beta}{\alpha} \int_{0}^{w} \zeta(\tau) d \tau}\right)
$$


where $g$ is the inverse function of $h=F^{\prime}$ on $\mathbf{R}^{+}$. Thus we obtained that (11) is satistied for $f(w)=\int_{0}^{w} f^{\prime}(\tau) d \tau$, where $f^{\prime}$ is given by $(21)$.

By changing the parameter $m \in(0, \infty)$ we can construct $f$ such that $f(1)$ is any positive number.

The proof of the theorem now will easily follow from applying the barrier $f(w)$ in the inner convex ring (10) with the parameter $m$ to be chosen such that

$$
f(1)=\min _{x \in B_{r_{D} / 2}\left(\left(0, \ldots, 0, r_{D}\right)\right)} u(x)>0 .
$$

Remark 7. Observe that if $f(w)$ is a sub-solution of (1) then in general we cannot say anything about the function $\alpha f(w)$, and we should construct the appropriate sub-solution by changing the parameter $m$ in (21).

Remark 8. If the boundary value of a non-negative $H$-harmonic function $u$ vanishes in a neighborhood of $y$ and the boundary $\partial D$ satisfies the outer convex $C^{1, D i n i}$ condition at $y$, then we can apply the super-solution barrier $f(1)-f(w)$ in the outer convex ring (see (10)), and obtain the Lipschitz bound

$$
u(x) \leq C M \operatorname{dist}(x, K),
$$

for $x \in B_{r_{D}}(y) \cap D$, where $r, C$ depending only on $D$ and $M=\max _{B_{r_{D}}(y)} u$.

Remark 9. One can make the condition (6) even weaker: there exists a constant $\alpha>0$ and a monotone increasing continuous function

$$
L: \mathbf{R}^{+} \rightarrow \mathbf{R}^{+}, \quad L(0)=0, \quad L(\infty)=\infty
$$

such that

$$
\int_{t}^{T} R(c(s) s) d s \geq \alpha \int_{L(t)}^{L(T)} R(s) d s .
$$

For functions $F$ satisfying (22) one will obtain

$$
f^{\prime}(t)=L^{-1} g\left(F^{\prime}(L(m)) e^{\frac{1}{\alpha} \int_{0}^{t} \zeta(\tau) d \tau}\right),
$$

where $g$ is the same as in $(21)$.

\section{Appendix: A comparison principle}

The comparison principle for $p$-harmonic functions is well known (see [HKM]), but for the general operator $\Delta_{H}$ we could not find a reference. Therefore we shall present a proof of this.

Theorem 2. Let $u$ be a weak solution of (1) and $v$ be its weak sub-solution in the domain $D$ with $C^{1}$ boundary. Further let $v \leq u$ on $\partial D$ in the sense of trace operator. Then $v \leq u$ in $D$.

Proof. Let us denote by $F^{*}$ the Legandre transform of $F$. Observe that $g(t)=$ $\left(F^{*}(t)\right)^{\prime}$ is the inverse function of the function $h(t)=F^{\prime}(t)$. By Young's inequality

$$
a b \leq F(a)+F^{*}(b)
$$

and the equality holds if and only if $b=h(a)$.

If $u$ is weak solution of (1) then from the convexity of $F$ it follows that

$$
\int_{D} F(|\nabla u|) d x \leq \int_{D} F(|\nabla w|) d x
$$


for any $w$ such that $u-w \in W_{0}^{1, p}(D)$. Otherwise

$$
\begin{aligned}
\int_{D} F(|\nabla(u+t(w-u))|) & \leq(1-t) \int_{D} F(|\nabla u|) d x+t \int_{D} F(|\nabla w|) d x \\
& <\int_{D} F(|\nabla u|) d x-\epsilon t
\end{aligned}
$$

for some $\epsilon>0$ and differentiating in $t$ we obtain

$$
\int_{D} H(|\nabla u|) \nabla u \nabla(u-w) d x<0
$$

which gives a contradiction after approximating $u-w \in W_{0}^{1, p}(D)$ by a test function $\phi \in C_{0}^{\infty}(D)$.

Let us now assume that $v \not \leq u$ and take as a test function $\psi=(v-u)^{+}$. By the definition of the sub-solution

$$
\int_{D} H(|\nabla v|) \nabla v \nabla \psi d x \leq 0
$$

Thus

$$
\int_{D_{1}} H(|\nabla v|) \nabla v \nabla v d x \leq \int_{D_{1}} H(|\nabla v|) \nabla v \nabla u d x
$$

where $D_{1}=\operatorname{supp} \psi \subset D$. Using now Young's inequality we obtain

$$
\begin{aligned}
\int_{D_{1}} H(|\nabla v|) \nabla v \nabla u d x & \leq \int_{D_{1}} h(|\nabla v|)|\nabla u| d x \\
& \leq \int_{D_{1}} F(|\nabla u|) d x+\int_{D_{1}} F^{*}(h(|\nabla v|)) d x .
\end{aligned}
$$

Since $h(t)=F^{\prime}(t)=t H(t)$, and Young's inequality is an equality for $b=h(a)$, we deduce

$$
\begin{aligned}
\int_{D_{1}} H(|\nabla v|) \nabla v \nabla v d x & =\int_{D_{1}} h(|\nabla v|)|\nabla v| d x \\
& =\int_{D_{1}} F(|\nabla v|) d x+\int_{D_{1}} F^{*}(h(|\nabla v|)) d x .
\end{aligned}
$$

By (23)-(25) we arrive at

$$
\int_{D_{1}} F(|\nabla v|) d x \leq \int_{D_{1}} F(|\nabla u|) d x
$$

where the inequality is strict unless $\nabla u=\nabla v$ a.e. in $D_{1}$; a contradiction in since $u-v \in W_{0}^{1, p}\left(D_{1}\right)$.

Acknowledgment. The authors would like to thank the Isaac Newton Institute for Mathematical Sciences, Cambridge, for support and hospitality during the program Free Boundary Problems and Related Topics where work on this paper was finalized. The first author is grateful to Stephan Luckhaus and Juan Luis Vazquez for inspiring discussions. 


\section{References}

[D] Dacorogna, B.: Introduction to the calculus of variations. - Imperial College Press, London, 2009.

[Dam] Damascelli, L.: Comparison theorems for some quasilinear degenerate elliptic operators and applications to symmetry and monotonicity results. - Ann. Inst. H. Poincaré Anal. Non Linéaire 15:4, 1998, 493-516.

[HKM] Heinonen, J., T. Kilpeläinen, and O. Martio: Nonlinear potential theory of degenerate elliptic equations. - The Clarendon Press, Oxford Univ. Press, New York, 1993.

[Hopf] Hopf, E.: A remark on linear elliptic differential equations of second order. - Proc. Amer. Math. Soc. 3, 1952, 791-793.

[L] Lewis, J. L.: Capacitary functions in convex rings. - Arch. Ration. Mech. Anal. 66:3, 1977, 201-224.

[LN] Lewis, J. L., and K. Nyström: Regularity of Lipschitz free boundaries in two-phase problems for the $p$-Laplace operator. - Adv. Math. 225:5, 2010, 2565-2597.

[Li] Lieberman, G. M.: Boundary regularity for solutions of degenerate elliptic equations. Nonlinear Anal. 12:11, 1988, 1203-1219.

[MPS] Manfredi, J., A. Petrosyan, and H. Shahgholian: A free boundary problem for $\infty$ Laplace equation. - Calc. Var. Partial Differential Equations 14:3, 2002, 359-384.

[RR] RaO, M. M., and Z. D. Ren: Theory of Orlicz spaces. - Marcel Dekker Inc., New York, 1991.

[RR1] RaO, M. M., and Z.D. Ren: Applications of Orlicz spaces. - Marcel Dekker Inc., New York, 2002.

[T] TolKsDorf, P.: On the Dirichlet problem for quasilinear equations in domains with conical boundary points. - Comm. Partial Differential Equations 8:7, 1983, 773-817.

[W] Widman, K.-O.: Inequalities for the Green function and boundary continuity of the gradient of solutions of elliptic differential equations. - Math. Scand. 21, 1967, 17-37.

Received 28 February 2014 • Revised received 17 November 2014 • Accepted 21 November 2014 